\title{
Development of haptic simulator for practicing the intraarticular needle injection under echography*
}

\author{
Ma de los Angeles Alamilla Daniel ${ }^{1}$, Richard Moreau ${ }^{1}$, Redarce Tanneguy ${ }^{1}$
}

\begin{abstract}
Intraarticular needle injection under ultrasound guidance is the most used method to provide pain relief in joints. To learn this process, medical students need to practice in order to master the manipulation of the syringe and the ultrasound probe at the same time. To offer a risk-free training for trainees, we present in this paper the development and design of a haptic simulator prototype. The models and methods used to render the forces involved are introduced, as well as the mechanical design and finally a validation test performed by our medical partner.
\end{abstract}

Clinical relevance - This simulator helps medical students to train intraarticular needle injection under ultrasound guidance using a haptic device to provide realism and immersion.

\section{INTRODUCTION}

The aspiration of joint effusion, soft tissue fluid collection, and injection inside the joint (knee, shoulder, hip, wrist, ankle) are common techniques used in various treatments and as routine procedures used by rheumatologists to relieve pain [1]. The palpation guided proximal interphalangeal is a traditional method used by doctors to help them to guide the needle into the joint. The ability to place a needle accurately within the joint space is quite essential to obtain a successful treatment. However, most rheumatologists ensure that without the help of imaging guidance, the correct positioning of the needle is quite challenging. Some studies reveal that under palpation guides, $59 \%$ of the cases were extra-articular which prevents extracting any fluid [2]. Therefore, methods based on ultrasound images tend to be more accurate during the puncture.

Intraarticular needle injection under ultrasound guidance is a technique used to achieve a successful positioning. The doctor uses an ultrasound probe to locate the needle tip placement in real-time during its insertion into the tissue [3]. This process is quite challenging as the doctor manipulates with one hand the needle trying to avoid touching a vein or cartilage (Fig. 1a) and with the other hand, the doctor holds the ultrasound probe to explore the joint and tries to keep the needle inside the 2D image (Fig. 1b). During the process, the doctor must focus his attention on observing the monitor instead of his hands' movements. Doctors need therefore to develop a motor visual-coordination dexterity that is only acquirable with practice to coordinate both syringe and ultrasound probe at the same time. Several health

\footnotetext{
*This work was supported by ANR(French National Research Agency) for financing SAMSEI project (ANR-11-1DFI-0034) under the supervision of Pr. X Martin

1 Authors are with lab. Ampère, CNRS UMR5005, Univ Lyon, INSA Lyon, Lyon, France richard.moreaulinsa-lyon.fr
}
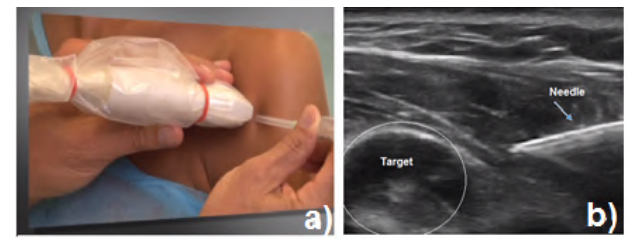

Fig. 1: Needle advance monitoring using ultrasoundguidance

authorities demand, from an ethical point of view, a way to practice without risk for the patients. One example is the French H.A.S. (Haute Autorité en Santé) who stated to medical students to "never make it the first time on a patient" [4]. A widely used solution to provide experience to medical students, is the use of haptic simulators, as they provide a safe way to practice several times the medical process without risking the patient's integrity. Although haptic simulators have been developed by several researchers and companies around the world, they still do not provide a fully immersive experience or realism. In the case of intraarticular needle injection under ultrasound guidance, current simulators fail to give the user the option to choose the needle's entry-point and rendering realistic forces that occur during the injection.

From this problematic, the SPARTE (Simulator of Puncture for ARTiculations under Echography) project aims to develop a simulator to offer new training methods for medical students. This simulator is divided into two main parts: the needle insertion simulator, and the ultrasound echography simulator.

In this paper, we presented the development of the needle insertion simulator for intraarticular needle injection. This simulator is focused on replicating the haptic forces while the needle punctures and cuts tissues. For rendering these forces, we implemented four mathematical models obtained from the literature and one original method. In collaboration with our medical partners, we designed a mechanical base that helps the medical students to perform the procedure in a more immersive and comfortable way.

This paper is structured as follows: in section II we show a brief state of the art of current simulators. In section III we introduce our needle insertion simulator and the methods used to render forces. The mechanical design, as well as the virtual environment, are then presented. Section IV is dedicated to the presentation of the results following tests with our medical partners. Finally, in section V we provide conclusions and perspectives about this work. 


\section{STATE OF ART}

In the literature, some simulators have been proposed for intraarticular needle injection. Forest et al. [5] proposed to use two identical haptic devices to achieve a simulator for ultrasound-guided needle puncture. In this configuration, one haptic device is used to represent the needle forces during the insertion and the other to simulate the ultrasound probe. They created a virtual environment where a $2 \mathrm{D}$ image ultrasound is displayed during the performance. This image is based on data obtained from real ultrasound images. Only one anatomy is thus available on this simulator which limits its application as a learning tool. Another drawback is due to the fact the simulator does not offer surface support on which the user can lie his hand to avoid trembling during the needle insertion. Another ultrasound-guided needle simulator is the one presented in [6]. The authors use a mannequin to represent the patient. The ultrasound probe and the needle have 3D position sensors. On a screen, a software recreates the ultrasound image, and for the force feedback, the needle has a slide mechanism that contracts itself when collisions occur with the plastic mannequin. This simulator allows to puncture of any part of the patient's body but does not constrain the position of the needle, allowing thus all displacements of the needle which limits the realism.

It exists other simulators [7], [8] but none of them completely fulfills the needs established by our medical partner, which are:

1) To allow the doctor to choose a needle insertion point in any place on the joint.

2) Once the insertion point is selected, the haptic interface must keep the same direction, and if it is necessary, the trajectory can be modified within the body if a prefixed maximum angle is not exceeded

3) When the needle tip is penetrating the body, different body stiffness must be rendered, according to patient's profiles and pathologists.

4) If the doctor stops his movement within the body, the needle must remain in the same position.

5) The simulator must have support for the hand, as doctors lay his/her hand in the patient's limb during the injection, to stabilize himself during the puncture.

Based on these criteria, we developed a simulator that satisfies them.

\section{NEEDLE INJECTION SIMULATOR}

The development of this needle injection simulator is divided into three parts. The first part is the study and selection of models and algorithms that are used for rendering forces during the insertion. The second part concerns the mechanical design. The last part focuses on the virtual environment on which the user can see its actions while interacting with the simulator.

\section{A. Rendering forces}

While needle pierces soft tissue, three stages can be distinguished [9]. Each stage generates a set of forces that need to be rendered through the haptic device to achieve enough realism.

1) Stage 1: Pre-puncture: This force is generated when the needle is pushing the surface of the tissue which becomes distorted without being piercing. This behavior is described as a viscoelastic interaction [10].

To render this force, we used the model proposed in [11], where authors obtained the numerical values by puncturing a bovine liver. The authors describe this force using a secondorder polynomial: the force increases steadily and then it sharply drops to indicate the puncture event [12].

2) Stage 2: Puncture: This force, denoted as cutting force, acts on the needle tip in the axial direction. Its intensity differs according to the various tissue layers as well as the shape of the needle tip. For this force, the "tracking wall" method is used [13]. This method consists of implementing a virtual wall that follows the needle's position, with a small position difference between them, to ensure a constant force during the injection. Once the needle stops its progression, the wall is smoothly updated to the needle's last position. It allows to replicate tissue behavior with a low rejection force.

To this force, a friction force can be added to increase realism. It acts along the side of the needle shaft in the axial direction. To reproduce it, we opted to use a realistic approach as in [14]. In this paper, the authors use the Lugre model which is a dynamic model based on the microscopic representation of irregular contact surfaces and elastic bristles. The authors obtain the parameters of the model by making a periodic penetration in a Gellan Gum with a needle attached to a 5-DoF manipulator.

Finally, the last force is added during this stage. This force, denoted clamping force, acts on the side of the needle shaft in the normal direction by the tissue that surrounds it and constrain the needle's movements. To implement this force, we opted to use a virtual fixture as in [15]. The virtual fixture provides a bilateral restriction that ensures the needle to move along the desired path. If the user attempts to deviate from this trajectory, the haptic device exerts a force in order to return on the initial trajectory.

All these different forces are shown in Fig 2.

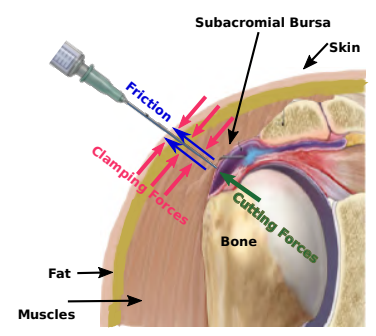

Fig. 2: Interaction forces during needle insertion.

3) Stage 3: Withdrawal: The third stage describes the force when the needle is withdrawn from the tissue. This force denoted withdrawn force, is opposite to the motion of the needle. Its behavior is similar to the puncture force. We, therefore, use the same implementation as the one described in the second stage. 


\section{B. Mechanical design}

The mechanical part of the simulator (Fig. 3a) consists of a Haption Virtuose 6D desktop haptic device, which has $6-\mathrm{DoF}$ motorized and offers a maximum force of $10 \mathrm{~N}$. The device is attached to a base and a support that represents the patient. To allow the haptic device's tool to penetrate the needle's support, an insertion point has been set to allow the tool to pass through it freely. To the tip of the haptic device, a mock needle mounted on a syringe has been attached to ensure realism.

\section{Virtual Environment}

A user-friendly interface was implemented using the framework CHAI3D [16]. The virtual environment consisted of a virtual articulation that helps the user to have more immersive experience due to its similarity to the real workspace (Fig. 3b). Users can rotate the virtual articulation freely and choose the point of insertion. For our tests, the chosen articulation is a shoulder, but it could be easily changed.
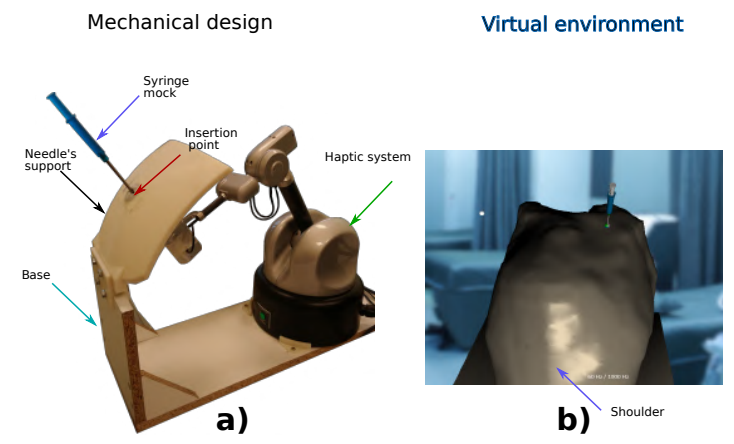

b)

Fig. 3: Test bench

Fig. 4 shows the global SPARTE simulator. The user can practice the intraarticular needle injection under echography. The needle's haptic feedback is provided by our system. For practicing the needle guidance, the user uses an ultrasound probe mock, and visualize a $2 \mathrm{D}$ image rendered in real-time by the software developed in [17].
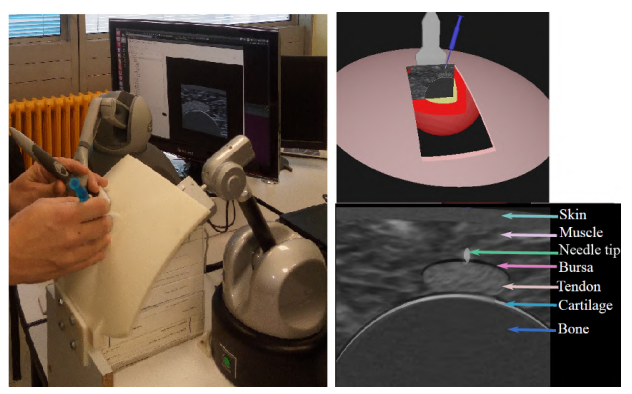

Fig. 4: SPARTE project simulator: haptic interface [13] and ultrasound images generator[17]

\section{TEST AND RESULTS}

To validate our simulator, we performed two preliminary tests that consisted of inserting the needle and trying to follow its trajectory towards the bursa, using the ultrasound probe mock. These experiments were dedicated to the validation of different forces (clamping, cutting, withdrawn and friction) during the injection. The values of these forces were established according to the literature [18] and determined empirically with our medical partner. The desired cutting forces used during the injection for each layer were $0.8 \mathrm{~N}$ (Skin), $1.5 N$ (Fat), $2 N$ (Muscle), $2.5 N$ (Bursa), $3 N$ (Tendon) and $4 N$ (Cartilage).

The first injection consisted of reaching the bursa and keep the needle visible all the time on the ultrasound image. The user intentionally missed the bursa in order to recreate a situation where it is necessary to change the trajectory of the needle. He thus withdraws its needle a few centimeters and then changes its orientation before piercing again the tissue in order to reach the bursa. The second injection consisted of puncturing and withdrawing the needle without changing its trajectory.

The corresponding needle tip trajectories in the simulator global framework are shown in Fig 5. On these figures, the clamping forces are also represented. As a reminder, these forces appear when the user does not follow its initial trajectory and they are applied along the normal direction of the needle shaft.

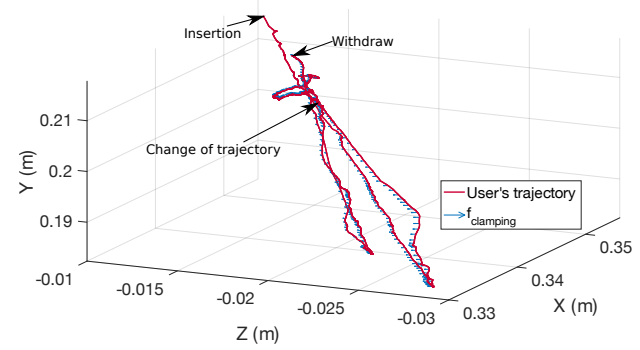

(a) First injection

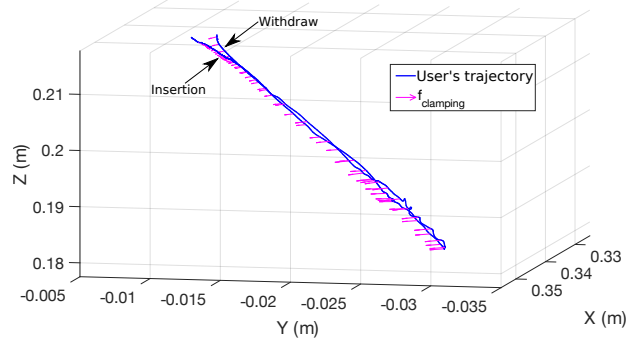

(b) Second injection

Fig. 5: Needle's trajectories

For the two injections, the cutting forces are shown in Fig.(6). When the user withdraws its needle, the same kind of force is calculated but in the opposite direction.

During the first injection (Fig. 5a), the user can feel the different cutting forces of each layer that the needle's tip pierces. When the user visualizes the bursa on the simulated 2D ultrasound image, he withdraws the needle in order to correct its orientation. When the user corrects the orientation of the needle, the puncturing is resumed, and he can feel the piercing of each layer again. When he visualizes the needle reaching the cartilage, he withdraws the needle completely 


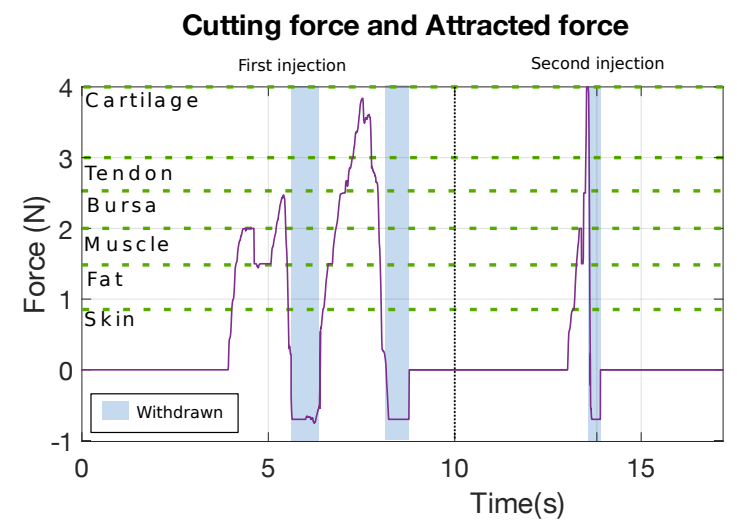

Fig. 6: Cutting forces and attracted forces

from the tissue.

In the second injection (Fig. 5b), the forces that the user feels are rendered in order according to the needle current position. As in this test, there is no needle's orientation correction, the user pierces all the layers until the cartilage, and then proceed to withdraw the needle completely.

In both injections, the user feels the withdrawn forces (blue zone in Fig. 6) when the needle is withdrawn, either to correct the needle's orientation or to end the process.

\section{CONCLUSIONS}

To train medical doctors to intraarticular needle insertion under ultrasound guidance, a simulator has been developed. It consists of a mechanical component which reproduces the involved forces during the needle insertion, the needle withdrawn, and the needle change of orientation. The second component developed by our partner consists of a real-time ultrasound images generator. The simulator has been tested and validated by our medical partner and according to her commentaries, the simulator provides a realistic sensation, which she considers good enough for introducing novice students to the desired procedure. Contrary to some simulators presented in the literature, this simulator offers a complete solution for training: support quite similar to real life, haptic feedback during needle insertion, real-time ultrasound image generator, data recording of the gestures in order to proceed to the evaluation.

Another advantage of our simulator is the possibility to customize the simulated patient and thus the difficulty: profiles (weight, height, age), different pathology, and joint.

Although the needle's insertion point is fixed, the virtual environment allows us to rotate and move the limb to reach any desired landmark. The immersion of the simulator is provided by the virtual environment and the syringe mock which was modeled based on a real one used for this process.

A campaign of measurement should be held soon to test with different expert doctors, and with some novices in order to evaluate if, the proposed simulator, helps to reduce the learning curve, and allows to gain experience faster, before practicing on real patients.

\section{ACKNOWLEDGMENT}

The authors would like to express their gratitude to the Consejo Nacional de Ciencia y Tecnologia (CONACyT) in Mexico for providing financial support to the PhD student and we would like to thank the ANR (French National Research Agency) for financing SAMSEI project(ANR-11IDFI-0034) under the supervision of Pr. X. Martin.

\section{REFERENCES}

[1] P. V. Balint, D. Kane, J. Hunter, I. B. McInnes, M. Field, and R. D. Sturrock, "Ultrasound guided versus conventional joint and soft tissue fluid aspiration in rheumatology practice: A pilot study," Journal of Rheumatology, vol. 29, no. 10, pp. 2209-2213, 2002.

[2] P. Peetrons and M. Court-Payen, "Ultrasound-Guided Musculoskeletal Interventional Procedures," pp. 385-398, Springer Berlin ISBN: 9783-540-49929-9, 2009.

[3] C. M. Sofka, A. J. Collins, and R. S. Adler, "Use of Ultrasonographic Guidance in Interventional Musculoskeletal," Journal of Ultrasound in Medicine, pp. 21-26, 2001.

[4] J.-C. Granry and M.-C. Moll, "État de l'art (national et international) en matière de pratiques de simulation dans le domaine de la santé," tech. rep., Haute Autorité de la Santé (HAS), 2012.

[5] C. Forest, O. Comas, C. Vaysière, L. Soler, and J. Marescaux, "Ultrasound and needle insertion simulators built on real patient-based data," Studies in health technology and informatics, vol. 125, pp. 136139, 2007.

[6] D. Magee, "A computer based simulator for ultrasound guided needle insertion procedures," IEE International Conference on Visual Information Engineering (VIE 2005), pp. 301-308, 2005.

[7] M. Raitor, J. M. Walker, A. M. Okamura, and H. Culbertson, "WRAP: Wearable, restricted-aperture pneumatics for haptic guidance," Proceedings - IEEE International Conference on Robotics and Automation, pp. 427-432, 2017.

[8] O. Goksel, K. Sapchuk, and S. E. Salcudean, "Haptic simulator for prostate brachytherapy with simulated needle and probe interaction," IEEE Transactions on Haptics, vol. 4, no. 3, pp. 188-198, 2011.

[9] L. Barbé, B. Bayle, M. De Mathelin, and A. Gangi, "Needle insertions modelling: Identifiability and limitations," IFAC Proceedings Volumes (IFAC-PapersOnline), vol. 6, no. PART 1, pp. 129-134, 2006.

[10] Y.C. Fung, Biomechanics. Mechanical Properties of Living Tissues. New York: Springer Science ISBN:978-1-4757-2257-4, 2 nd ed., 1993.

[11] C. Simone and A. M. Okamura, "Modeling of Needle Insertion Forces for Robot-Assisted Percutaneous Therapy," in Proceedings of the 2002 IEEE International Conference on Robotics \& Automation, no. May, pp. 2085-2091, 2002.

[12] N. Abolhassani, R. Patel, and M. Moallem, "Needle insertion into soft tissue: A survey," Medical Engineering and Physics, vol. 29, no. 4, pp. 413-431, 2007.

[13] M. Alamilla D, R. Moreau, and T. Redarce, "A new method to render virtual walls for haptic systems: Tracking wall. Application to needle insertion simulation," in ICVARS'19 Proceedings of the 2019 3rd International Conference on Virtual and Augmented Reality Simulations, pp. 33-38, 2019.

[14] A. Asadian, R. V. Patel, and M. R. Kermani, "A distributed model for needle-tissue friction in percutaneous interventions," Proceedings - IEEE International Conference on Robotics and Automation, pp. 1896-1901, 2011.

[15] R. Kikuuwe, N. Takesue, and H. Fujimoto, "Passive virtual fixtures based on simulated position-dependent anisotropic plasticity," in Proceedings - IEEE International Conference on Robotics and Automation, pp. 3263-3268, 2007.

[16] F. Conti, F. Barbagli, R. Balaniuk, M. Halg, C. Lu, D. Morris, L. Sentis, J. Warren, O. Khatib, and K. Salisbury, "The CHAI libraries," in Proceedings of Eurohaptics 2003, pp. 496-500, 2003.

[17] C. Barnouin, F. Zara, and F. Jaillet, "A real-time ultrasound rendering with model-based tissue deformation for needle insertion," in 15th International Conference on Computer Graphics Theoryand Applications, GRAPP 2020., (Valleta, Matal), 2020.

[18] A. Gordon, A. C. Barnett, I. Kim, and J. Z. Moore, "Needle insertion force model for haptic simulation," ASME 2015 International Manufacturing Science and Engineering Conference, MSEC 2015, vol. 2, pp. 18-20, 2015. 